\title{
Running Infiniband on the Cray XT3
}

\author{
Makia Minich \\ Oak Ridge National Laboratory \\ Oak Ridge, $\mathrm{TN}$ \\ minich@ornl.gov
}

Keywords: Cray, XT3, infiniband, Voltaire, linux, OFED, OpenFabrics

\begin{abstract}
In an effort to utilize the performance and cost benefits of the infiniband interconnect, this paper will discuss what was needed to install and load a single data rate infiniband host channel adapter into a service node on the Cray XT3. Along with the discussion on how to do it, this paper will also provide some performance numbers achieved from this connection to a remote system.
\end{abstract}

\section{OVERVIEW AND GOALS System Layout}

Since a discussion of the Cray XT3 architecture is beyond the scope of this document, we are going to focus on the overall layout of the systems used in our test. Figure 1 shows the extremely basic overview of the connections between our systems. (If you would like more specifics on the Cray XT3 architecture, visit the Cray website ${ }^{1}$ which has a lot of useful marketing media that provides a good overview.)

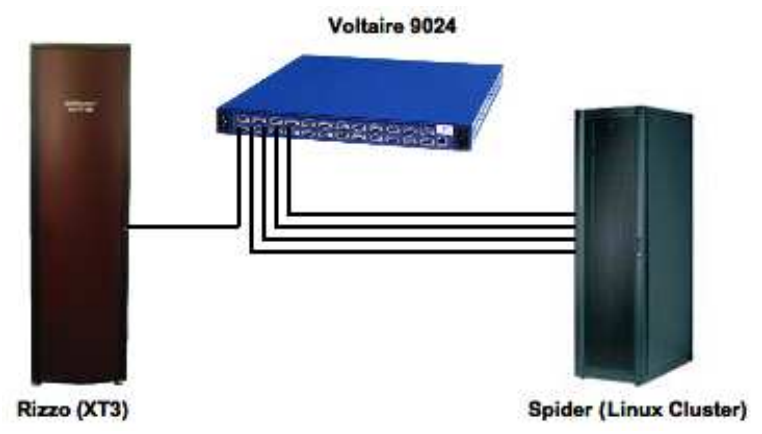

Figure 1. System Layout

Rizzo is a single rack of XT3 hardware comprised of 14 IO nodes (7 IO modules) and 68 compute nodes (17 compute modules). Each of these IO nodes has a single $133 \mathrm{MHz}$ PCI-X available for an expansion card. For this testing, we have placed a dual-port, single data rate (SDR), 128MB Voltaire host-channel adapter (HCA) into one of the IO nodes and connected it to a Voltaire 9024 (24-port SDR infiniband

\footnotetext{
${ }^{1}$ http://www.cray.com
}

switch). While it would be more preferential (for testing as well as moving forward) to have more than one HCA in Rizzo, at the time only one was installed.

On the other end, we have Spider which is an x86_64 based linux cluster. While Spider has a large number of nodes available, for this testing only four nodes were used. Each of the nodes have a dual-socket dual-core 2.2GHz AMD Opteron with an 8-lane PCI-Express based Voltaire 4x SDR HCA.

The Voltaire 9024 and Spider are co-located, which allows us to use a standard CX4 infiniband cable (1 meter lengths) between the nodes of Spider and the Voltaire switch. Rizzo happens to be a larger distance away (around 23 meters), so we needed to use Emcore's SmartLink QTR3400²-a CX4 to fiber converter-to allow us to run a longer fiber connection between Rizzo and the Voltiare 9024.

Normally, when someone talks about infiniband and clusters, they are talking about using it as a high-performance interconnect within a cluster. But, as you can see, in this testing we're using it to bridge two (or more) clusters together so that we can provide a fast data-movement path between the multiple clusters.

\section{Operating System Software \\ Operating System}

To avoid delving to deep into the intricacies of the XT3 software stack, we are going to focus on the two main pieces that we need to be aware of. The IO nodes (and any type of interactive node on the XT3) run diskless with a SuSE-derived base OS (currently based on SuSE Enterprise 9). The compute nodes, on the other hand, run Catamount which allows the compute nodes to boot a micro-kernel and an application. ${ }^{3}$ This allows the compute nodes to spend all of their cycles running the application which can help to reduce OS jitter. Spider, being a standard linux cluster, is running RedHat Enterprise Linux Workstation release 4 update 3. The system breakdowns can be seen in table 1 .

From the OS Comparison table we see that a kernel version is mentioned for the Catamount nodes. While one can't easily type uname $-r$ on the command line of the compute node (primarily due to the lack of any user-level interaction), there is an actual kernel version associated with that boot (hence

\footnotetext{
${ }^{2}$ http://www.emcore.com/assets/fiber/pb.QTR3400_\&_QTR3432.200412-12.Emcore.pdf

${ }^{3}$ http://www.cray.com/downloads/Cray_XT3_Datasheet.pdf
} 
Table 1. OS Comparison

\begin{tabular}{||l|l|l|}
\hline System & OS & Kernel Version \\
\hline Rizzo & UNICOS 1.4.19 & \\
$\bullet$ IO Nodes & SuSE Enterprise 9 & $2.6 .5-7.252-\mathrm{ss}$ \\
$\bullet$ Compute Nodes & Catamount & $2.6 .5-7.252-n i$ \\
\hline Spider & RedHat Enterprise Linux Workstation 4 update 3 & 2.6.9-42.EL_lustre.1.4.7smp \\
\hline
\end{tabular}

the $-\mathrm{ni}$ suffix in the table). This kernel is encapsulated in the stage 2 . sf file, which is created by the build process for the XT3 software stack.

\section{Infiniband Stack}

The OpenFabrics Alliance 4 recently began distributing an enterprise version of their stack. Created through a collaboration between different infiniband vendors and opensource commutinty contributors, the OpenFabrics Enterprise Distribution (OFED) is touted as the stable and supported opensource infiniband stack. While development is still ongoing for the main OpenFabrics software branch, the OFED stack takes snapshots in time, to create a supported product for the infiniband community. These releases supply an easy to build and install framework which allows users to start utilizing their infiniband interconnect regardless of what vendor and OS stack is loaded on the system. By unifying the infiniband stack, it has become easier to manage software revisions on multiple platforms as well as provide consistent API's for interconnect development on these platforms.

Our testing is focussing on OFED 1.0.1 which contains some amount of support for all of the kernels involved in our testing. While normally we would build all of the tools associated with the infiniband stack, our system layout precludes us from needing things like MPI. More importantly, we're going to need IP-over-IB (IPOIB) for standard ethernet connections, remote-DMA access to pass large amounts of data across the interconnect, and the sockets direct protocol (SDP) to efficiently encapsulate IP traffic into IB traffic.

\section{Test Suite}

The following tests were used to determine not only the functionality of the infiniband connection but also to graphically plot the performance. Because of the nature of our system (which is described in more detail in sections and), we can only focus on RDMA and IP based tests. As a side effect, though, this combination will also allow us to test SDP (sockets direct protocol) over the IP interface.

\section{RDMA Tests}

Provided as a default functionality test by the OpenFabrics Enterprise Distribution, ib_rdma_bw and ib_rdma_lat

\footnotetext{
${ }^{4}$ http://www.openfabrics.org
}

(RDMA bandwidth and latency respectively) allow us to measure the total throughput we could expect from the hardware (removing any constraints that the higher level infiniband protocols would impose).

The RDMA tests default to running at one packet size (65 kilobytes for ib_rdma_bw and 1 byte for ib_rdma_lat), so a script was needed to allow us to see what the trends are for multiple packet sizes. Listing 1 shows the script used to allow us to test from 2 to $2^{23}$ bytes for bandwidth and 2 to $2^{8}$ bytes for latency.

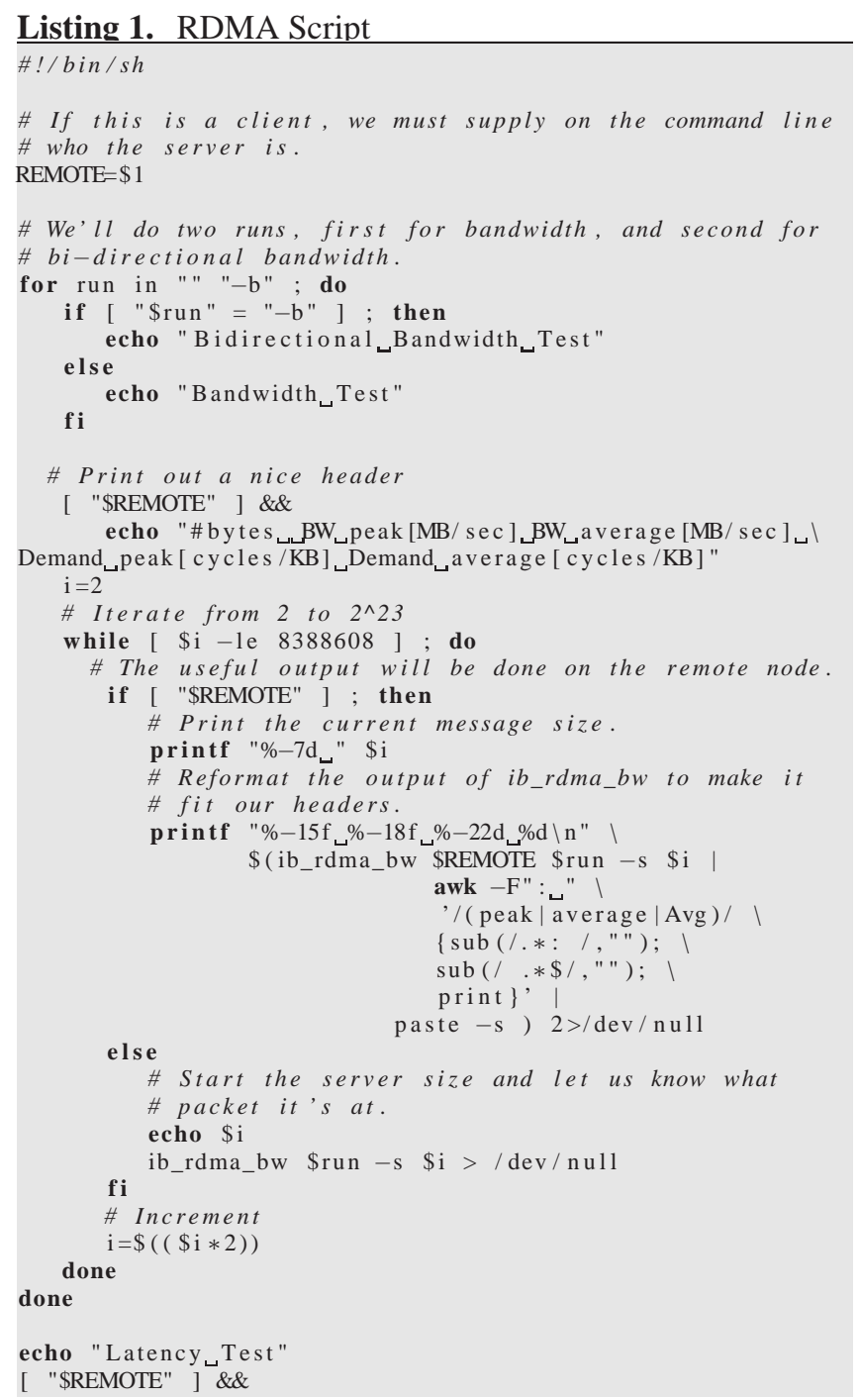




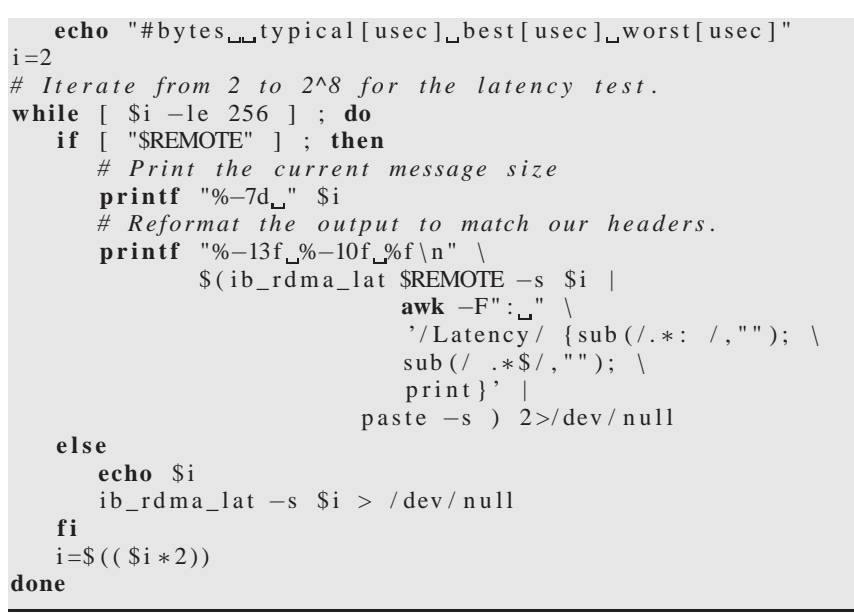

Plots of the output from this script are shown in the later figures.

\section{NetPIPE}

NetPIPE $^{5}$ is another bandwidth and latency measurement tool. While it does typically use MPI over Infiniband (or any other high performance interconnect), NetPIPE can also utilize tcp-based connections, which allows us to test IP-overIB (IPOIB) connections. NetPIPE performs a ping-pong style transfer to measure the transmission rates, and then outputs a table of latency and bandwidth measurements for a range of packet sizes.

Because of the nature of the TCP connections used by NetPIPE, we were easily able to use these same tests to measure the performance of SDP over the infiniband connection. By using LD_PRELOAD to load the libsdp . so libraries, we were able to use the same NetPIPE binary to test both standard TCP connections as well as SDP connections.

\section{Iperf}

Iperf $^{6}$ is another tool that attempts to measure maximum TCP bandwidth. This is a fairly standard network performance test and is supplied here just as an added comparison. Because of the TCP nature of this binary, we are able to again use LD_PRELOAD to load the libsdp . so libraries and thereby run Iperf over an SDP connection between nodes.

\section{GETTING INFINIBAND ON THE XT3}

On a normal cluster, such as Spider, building and loading the OFED stack is a relatively easy process. You can easily follow the instructions provided by the OFED release documentation to get things up and running. Life is a little bit different on the XT3 though, as there are a few caveats to keep in mind. The first is that we will only be affecting the IO

\footnotetext{
${ }^{5}$ See URL http://www.scl.ameslab.gov/Projects/NetPIPE

${ }^{6}$ See URL http://dast.nlanr.net/Projects/Iperf
}

nodes on the XT3, the Catamount nodes will need to rely on routing over Portals to utilize the infiniband connection (such as for lustre). The second is the limitations set out in the kernel provided by Cray. Because the XT3 is a fully supported platform, Cray makes specific decisions about what is made available in the kernel and what is available in the hardware. This is made painfully obvious when you attempt to build and load the OFED stack only to receive the dreadful unknown symbol errors.

\section{Kernel Changes}

When we were initially bringing up infiniband on the XT3, we required a couple changes to the default running kernel on the IO-nodes. Two symbols that were not exported by the kernel which OFED relies on; bad_dma_address and dev_change_flags. Applying the following patch to the IO-node kernel source, addresses this problem:

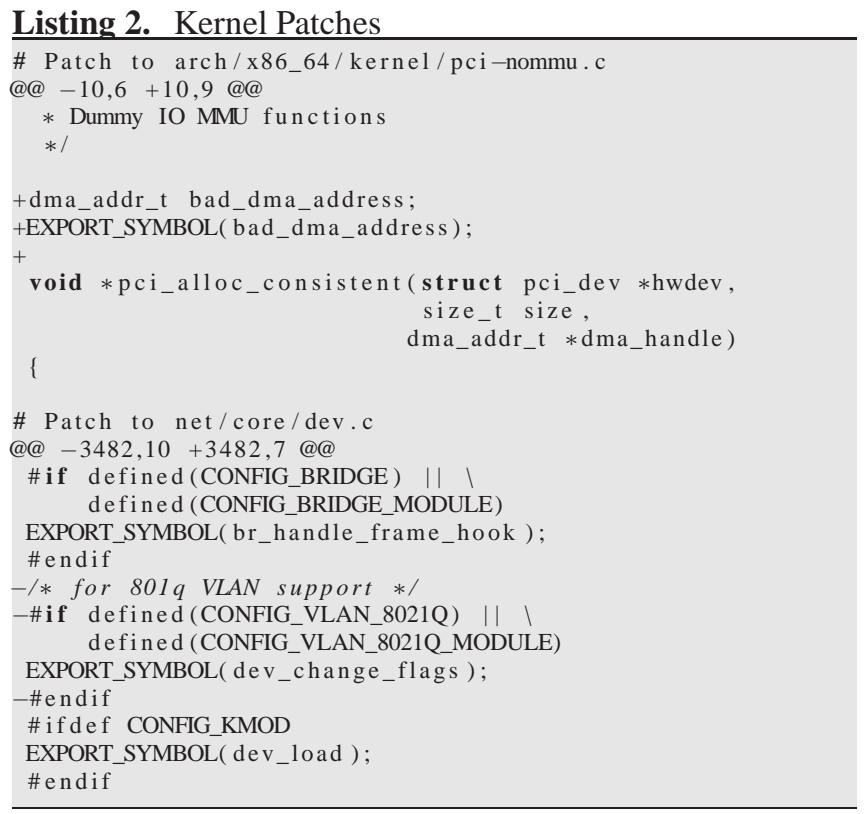

At this point, we are able to rebuild the kernel. We booted onto this kernel to make sure that everything was working properly. In order to build the OFED modules (covered in the next section), we used this modified source (rather than utilizing the kernel headers provided by the installed XT3 software).

Later versions of the XT3 kernel (starting with Unicos release 1.5 ) actually now have these patches incorporated. This makes building and running the OFED stack much easier in the long run. No longer do we need to rebuild the kernel, nor do we actually need the source code to build the OFED modules (instead we are able to utilize the header files located in /opt/xt-os/default). 


\section{Building and Loading OFED}

Once we had a working kernel, we proceed to building the OFED stack. To avoid kernel versioning mismatch errors, it is important to keep an eye on the gcc versions throughout this process. First, we need to make sure and build OFED with the same gcc version that the running kernel was built with (e.g. if the kernel was build with gec-3.2, you need to build the modules with gcc-3.2). As an added bonus, a lot of the OFED tools fail to compile with gec-3.2 and would prefer to be built with gec- 3.4 or higher. For this reason, we build the modules first and then the rest of the stack later. This is easily done by changing the ofed. conf file to first build the modules and then modifying it later to build the userspace tools.

At this point, we found that a change was needed to the OFED source code because it was found that the OFED stack didn't seem to recognize the XT3's kernel as a proper kernel value, and therefore didn't apply any of the needed patches to get things working. So, it was needed to decompress the openib-1.0.1.tgz source file, apply the patch in listing 3 and then re-compress.

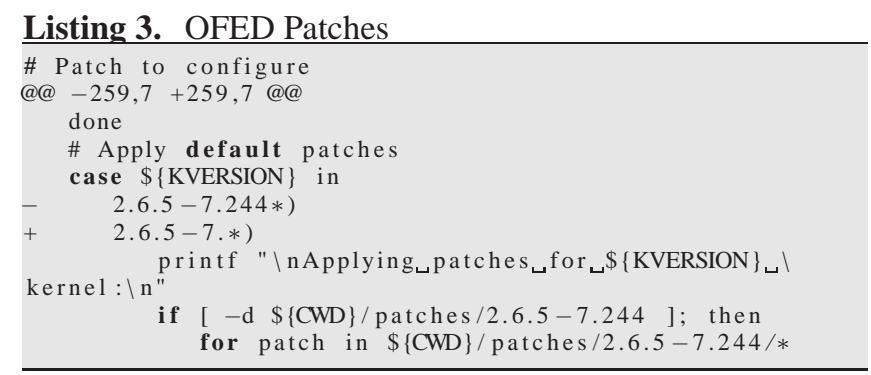

When all was said and done, we ended up with a few RPM's that we could then install into our IO node image and be off and running, configuring the system just like any other SuSE based image.

\section{PERFORMANCE OF INFINIBAND ON THE XT3}

After successfully getting the infiniband connection up and running on the XT3, we were able to measure the actual performance of the infiniband link. Because of our system architecture, there is a limit in the types of tests that could be run. In then end, though, we should be able to get a clear picture on what kind of performance we can achieve.

For each of these tests, we will be using a node from Spider (spider-server) as our server node. The client nodes, spiderclient and rizzo-io, will each connect to spider-server to perform the test. While it isn't preferential to mix the architectures (really, we should have two Rizzo nodes interacting) using the same server node for both tests at least gives a good starting point.

\section{RDMA Tests}

First, we'll start with the RDMA tests, which should give us a good baseline of the overall performance of the infiniband interconnect. For this test, we will be running a server on spider-server with clients on rizzo-io as well as spider-client. Figure 2 shows the results of this test.
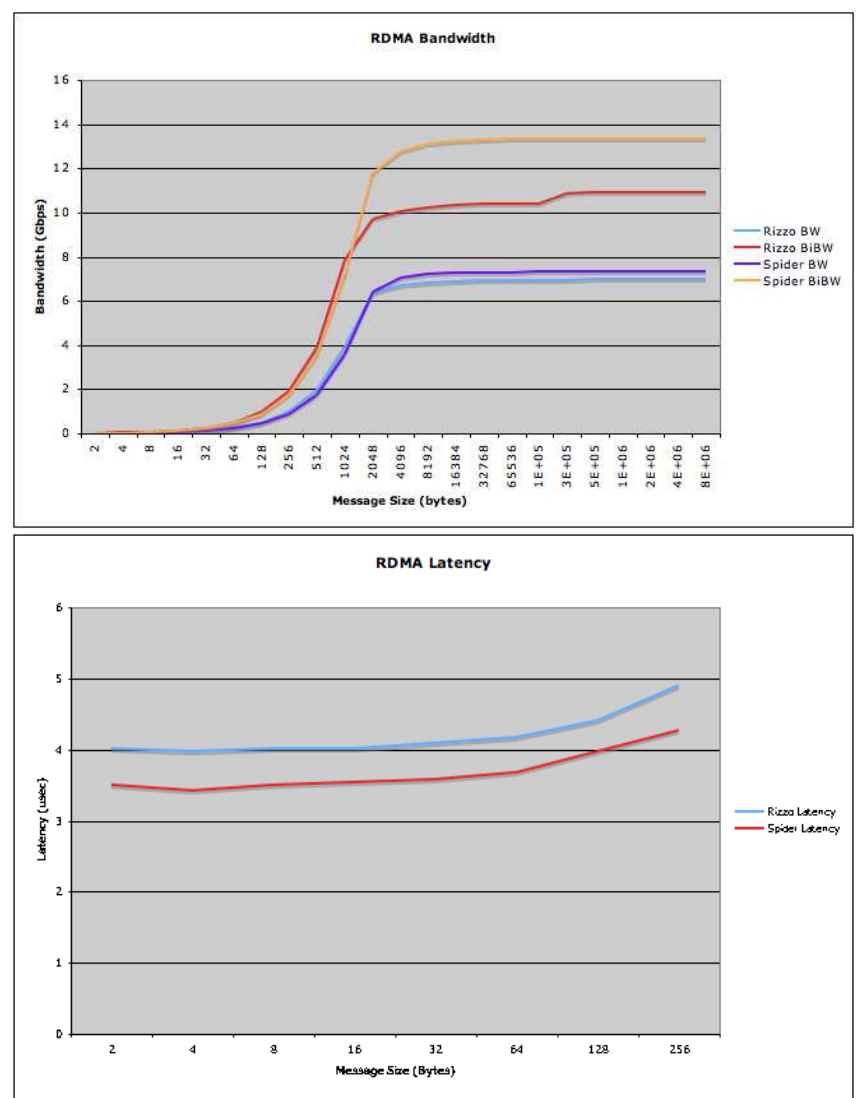

Figure 2. RDMA Performance

Right away one can see the benefits of the PCI-Express bus (specifically with the bidirectional tests), with about a $300 \mathrm{MB} / \mathrm{s}$ difference between Rizzo and Spider. But, there is something very interesting to pay attention to. The $133 \mathrm{MHz}$ PCI-X bus has a theoretical peak of $1 \mathrm{~GB} / \mathrm{sec}$, and Rizzo is performing at about $900 \mathrm{MB} / \mathrm{s}$, so unidirectionally we are able to achieve close to the same rates as the PCI-e links (many PCI-X based systems are still utilizing the $100 \mathrm{MHz}$ PCI-X bus, which would have caused a peak limit at about $800 \mathrm{MB} / \mathrm{sec})$.

The latency difference between the two systems may be a function of the bus differences, but more than likely this is due to the HCA hardware. There are a number of differences between the PCI-X release and the PCI-e release of the Voltaire $\mathrm{HCA}$, and one of the advancements has been in dealing with reducing the length of the path the packet needs to take in 
getting out of the HCA.

\section{NetPIPE}

Now that we've seen the raw bandwidth that should be available, we can now shift our focus to TCP based bandwidth. If we were to use something standard, such as NFS or SCP, over the infiniband connection, we would be relegated to the IP-over-IB interface. It is no secret that current IPoIB performance leaves a lot to be desired, but it is still useful to see what we can expect from IPoIB if we need to use it. As a side effect of this, we are also able to take a look at SDP performance (which basically encapsulates the TCP traffic and re-routes it to the RDMA level). While still in heavy development, SDP is already proving to be quite useful. Figure 3 shows the results of our testing.

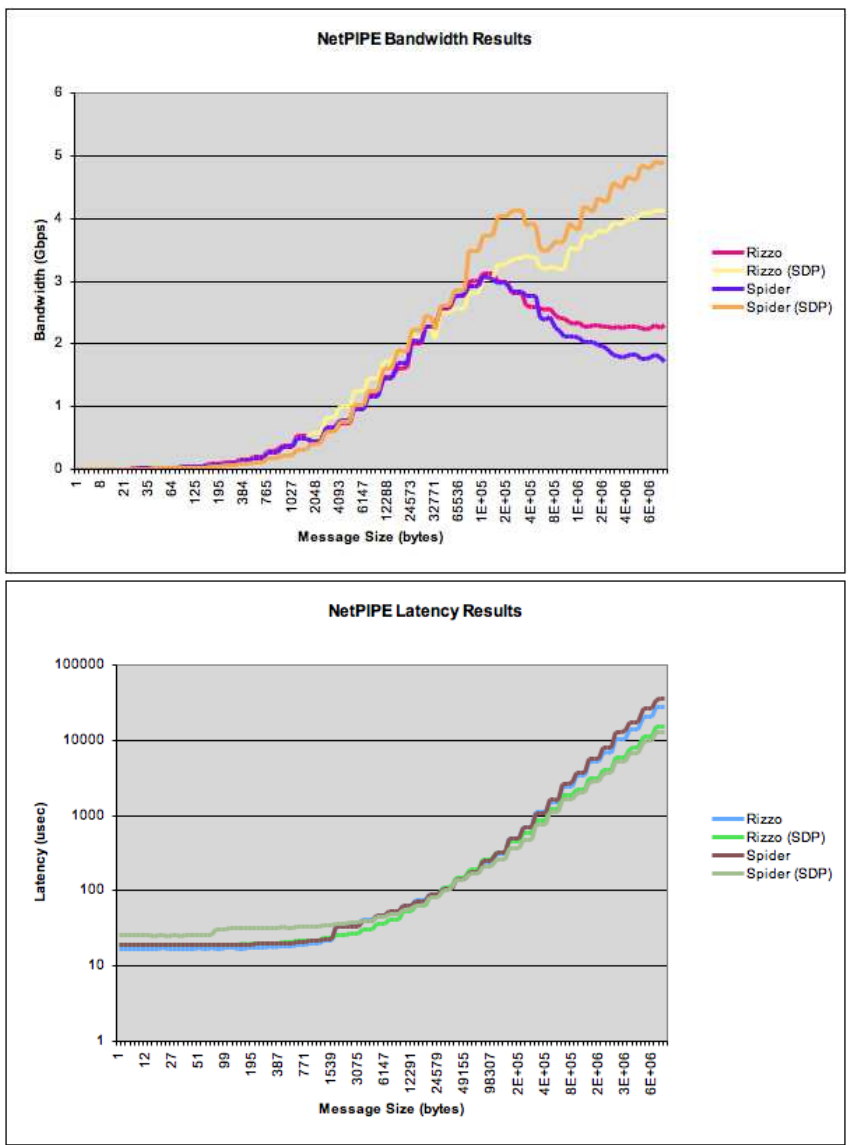

Figure 3. NetIPIPE Performance

On first glance, one can clearly see the primary benefit of SDP over the standard IPoIB interface. Where the SDP is concerned, one can also see the effect of PCI-e versus PCI-X. Oddly, though, in the IPoIB performance, Rizzo (with PCI$\mathrm{X}$ ) outperforms Spider. While we don't have any quantitative data to explain this, a possible explanation is the maturity of the PCI-X HCA compared to the PCI-e HCA (overall, more time has been put into the development of the firmware for the PCI-X HCA, which could lead to more time available to improve the IPoIB performance). The results from latency aren't overly surprising.

Another interesting point to mention is the comparison of the peak on SDP compared to the RDMA peak from the previous section. For RDMA, we're seeing about 7 to $7.5 \mathrm{Gbps}$ for a unidirectional stream, while we're only seeing between 4 and 5 Gbps from SDP. As more development continues on the SDP drivers, we should hopefully see these numbers improve.

\section{Iperf}

Our last test is just a quick Iperf run to verify the peak bandwidth that we're seeing with both IPoIB as well as SDP. Figure 4 shows the results.

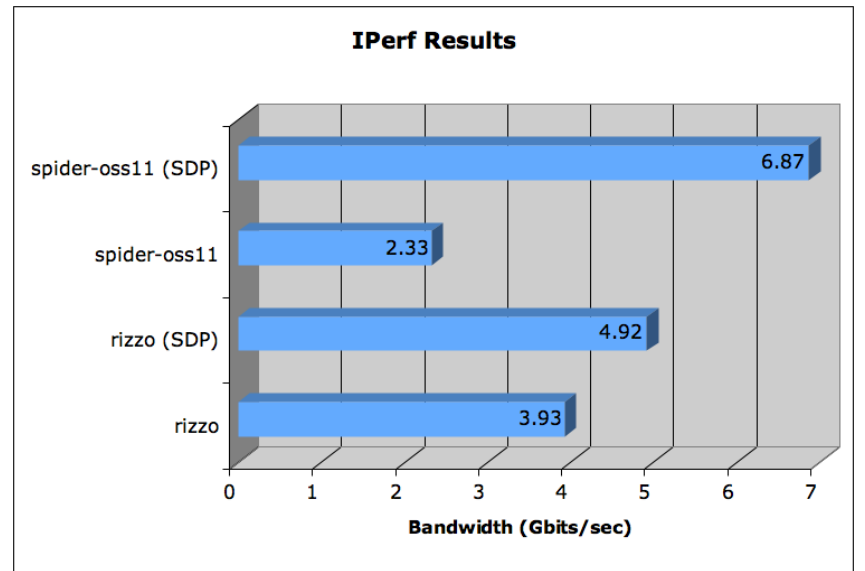

Figure 4. Iperf Performance

Again, we see the oddity of Rizzo besting Spider on straight IPoIB performance. Overall, though, we are not seeing anything surprising from these results and can feel a little more confidence in our ability to use the infiniband connection.

\section{CONCLUSIONS}

After a few gotchas in the initial attempts at bringing up infiniband on the XT3, we think that this shows quite well that it can be done both functionally and effectively. These first steps open the door to being able to provide another highspeed data movement path off the XT3. With this new ability, we could effectively, for example, utilize the infiniband interconnect as a storage network using SRP, iSER or even using Lustre ${ }^{7}$ over infiniband to move large data sets from the XT3 to a centralized Lustre filesystem. Though there is

\footnotetext{
${ }^{7}$ http://www.lustre.org
} 
a large amount of work still to be done to raise non-RDMA performance closer to RDMA levels, the door is at least open for the infiniband world to include the XT3.

\section{ACKNOWLEDGEMENTS}

The author would like to thank Don Maxwell from ORNL for putting up with innappropriately timed full system crashes and a ton of incessant XT3 questions. Also, we'd like to thank Andrew Lehtola from Cray for pushing our kernel changes through the management chain and into the real release. 